\title{
BURNS OF THE HAND *
}

\author{
BY
}

\section{P. H. JAYES}

\section{From the E.M.S. Plastic Surgery Centre, East Grinstead}

It is incumbent upon the industrial medical officer to acquaint himself fully with the modern treatment of burns, and to pay special attention to burns of the hands. Burns of the exposed areas of the body, especially the hands, frequently occur among factory workers and it is of paramount importance that these cases should receive expert treatment from the earliest stages. In many instances only a small percentage of the body surface is involved and there is very little disturbance in the general condition of the patient. But if the hands are affected vital issues are at stake. Errors of treatment may result in persistent stiffness, contractures, and permanent disablement; whereas proper care and attention can restore full function in the majority of cases even when the skin of the entire hand is destroyed.

In recent years many different methods have been advocated for the treatment of burns and fantastic claims have been made for a wide variety of lotions and ointments. It must be realized that successful results are dependent on close observance of the fundamental principles of wound treatment rather than on the application of a specific chemical substance. The choice of dressing for a burn varies under different circumstances but provision must always be made for free surgical drainage and the early separation of dead tissue. Furthermore, the technique adopted must aim at the control of infection combined with the preservation of function in joints and tendons.

First- and second-degree burns heal satisfactorily with almost any method of treatment, but thirddegree cases present many difficulties and are the crux of the whole problem. In these cases skin replacement is perhaps the most important single factor in treatment. If a burn results in a loss of skin on the hand or fingers extending over an area of more than a few square centimetres, a skin graft must be applied at the earliest opportunity to ensure a full functional result. Unless the skin which has been destroyed is replaced by a graft, healing can take place only by epithelium gradually growing in from the edges of the affected area until the whole surface is covered. This is a slow process and inevitably results in contractures and crippling deformity. Any method of treatment which is

* A paper read at a meetin of the Association of Industrial Medical Officers, January 22nd, 1944. advocated for third-degree burns must be regarded with suspicion unless it encourages the removal of sloughs and enables raw surfaces to be grafted successfully at an early stage.

Industrial burns of the hand are commonly localized, in contra-distinction to war burns which usually involve the entire hand. Many procedures which are advocated for encircling burns must be modified when the localized type is under consideration. Prognosis for function is dependent on the distribution of the burn as well as on the extent and depth of tissue destruction. Burns which affect the palm and flexor surfaces of the fingers are generally more serious than burns confined to the dorsum of the hand.

\section{First-Aid Treatment}

If the burn is of sufficient severity to necessitate hospital treatment, first-aid measures are confined to a minimum. The affected areas are covered with sterile gauze dressings (or clean linen, if sterile dressings are not available) and the patient transported to hospital as quickly as possible. In this country the patient can always be admitted to hospital within an hour or two of the accident and there is no indication for elaborate first-aid treatment. Attempts at cleaning or the application of ointments under such conditions increase the risks of infection and serve no useful purpose.

\section{Treatment on Admission to Hospital}

The patient must be examined and the treatment carried out under full aseptic conditions. Under morphine analgesia the affected areas are thoroughly but gently cleansed and for this purpose 1 per cent. 'Cetavlon' solution (cetyl-trimethyl-ammonium bromide) is most useful. Cetavlon is a detergent antiseptic solution which has considerable bactericidal power, and it deals effectively with any pathogenic organisms which are present on the surface of the burn or surrounding skin. Blisters are opened and loose epithelium carefully trimmed away with scissors. The burn is covered with a single layer of tulle gras, and them gauze wrung out in normal saline is applied and kept in place with bandages. The fingers must be dressed individually, opposing raw surfaces being kept apart by the dressings. 
This treatment should be carried out quickly with as little exposure of the burns as possible. General anaesthesia is usually unnecessary and should be employed only in exceptional circumstances, for example when required for associated injuries.

\section{Subsequent Treatment}

1. Dressings. If the general condition of the patient is poor the initial dressing is sometimes allowed to remain in position for three to four days but thereafter the dressings are changed twice daily. The outer layers of gauze are removed and the hand is immersed in normal saline at $100^{\circ} \mathrm{F}$. If the burn from the bath and a dressing of tulle gras and gauze wrung out in saline again applied.

2. Splinting and Elevation. With burns of the hand oedema is a prominent feature in the first few days and it is necessary to elevate the limb until the oedema has subsided. The hand is placed on a splint (plaster-of-paris or malleable metal) and the splint suspended from a Balkan beam to give the required elevation. The hand is splinted in the position of function, but this position will vary according to the distribution of the burn. When the entire hand is affected the fingers are placed in half flexion at the metacarpophalangeal and inter-

Right Hand:

1

2

3

4

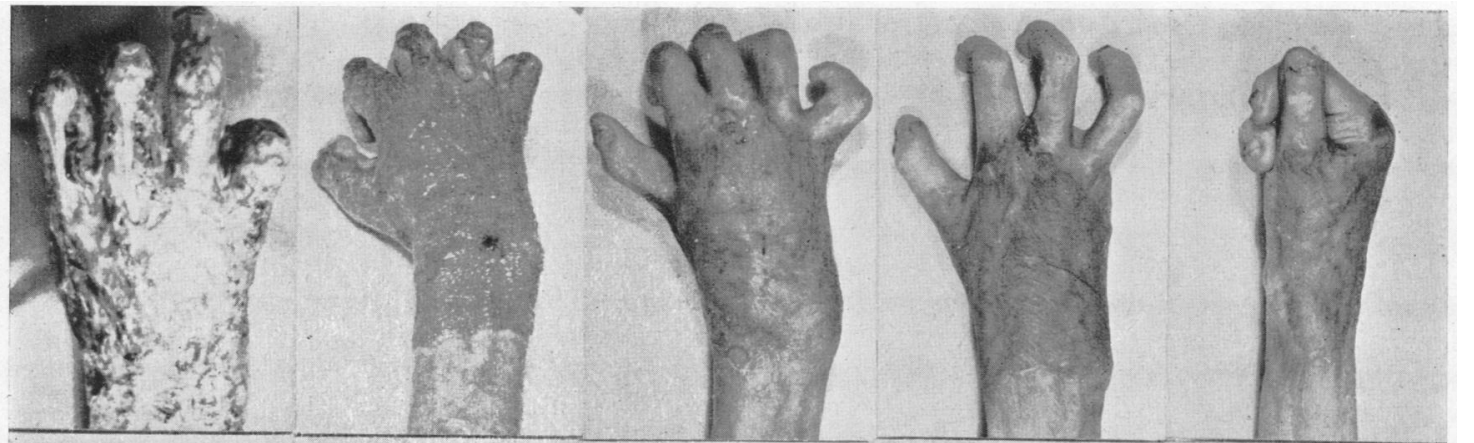

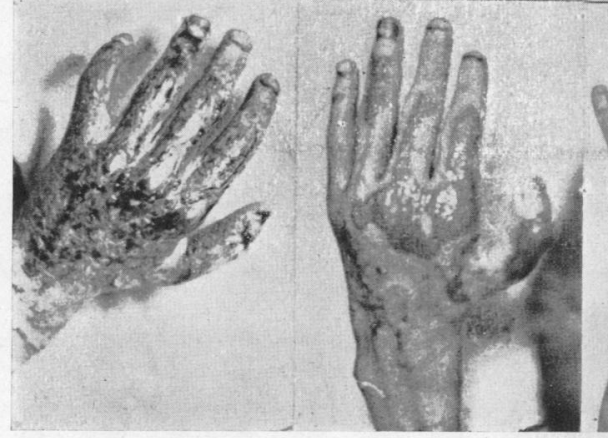

1

2

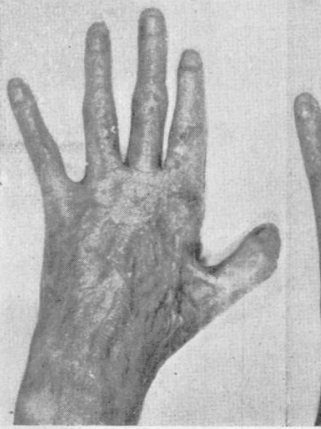

3

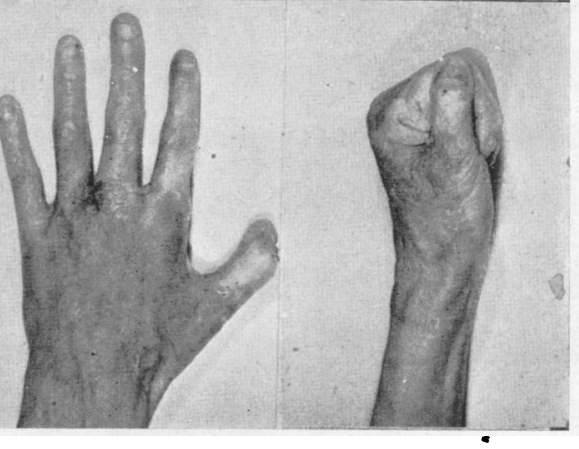

4 is confined to the hands, arm baths are used but when the burn also involves other parts of the body a total immersion:bath is more convenient. In the bath the gauze and tulle gras float away from the raw surfaces and the patient suffers very little discomfort from removal of the dressing. Epithelial debris and grease tend to collect at the margins of the burn and gentle swabbing is required to keep the whole area scrupulously clean. The time of immersion varies with the individual case, but after approximately 30 minutes the hand is withdrawn phalangeal joints, the tip of the thumb being in apposition with the index finger. When the burn is confined to the flexor surface the fingers and wrist are placed in the extended position. Great attention must be paid to these details for if any stiffness occurs the disability will be minimal if the hand has been treated in the position of function.

3. Movement. It is advisable to keep the fingers at rest until the oedema has subsided, but throughout the remaining period of treatment much emphasis is placed on active movement. The saline 
bath is a great help in the preservation of function as finger exercises can be performed with little discomfort while the hand is immersed in the saline.

In some third-degree burns the extensor tendons are exposed in the fingers, and occasionally the tendons are destroyed over the proximal interphalangeal joints. Division of the extensor expansions at this point results in exposure of the joints and ankylosis usually occurs later. In these cases it is very important to splint the fingers in the position of function, otherwise they become fully flexed owing to the unopposed-action of the flexors and ankylosis takes place in the flexed position with disastrous consequences from the functional standpoint.

Complications of this type may necessitate splinting for an extended period but the average burn of the hand is only immobilized for two or three days while the oedema is subsiding; the patient is then encouraged to move his fingers as much as possible. With this regime 'the frozen hand' is never seen and final healing is frequently coincident with a full range of movement in the fingers.

4. Preparation of Raw Surfaces for Grafting. One of the advantages of the saline method is that sloughs separate rapidly so that most third-degree burns are ready for grafting by the twenty-first day. Grafts cannot be applied until all dead tissue has been removed and healthy granulations are present. The granulations should be flat, firm and pink in colour with the epithelium growing in rapidly at the edge of the raw area. If they are oedematous, hypertrophic and spongy it is useless to apply grafts as these will float off in the pus which exudes from the surface. The bacteriology of the wound is determined and efforts are made to control infection before operation is undertaken. The organisms most frequently present are haemolytic streptococci, staphylococci, B. pyocyaneus and B. proteus. Streptococcal infection is the most frequent cause of failure of grafts. The local application of sulphanilamide powder to the burn is often successful in getting rid of haemolytic streptococci, but some strains of this organism are resistant to the drug. Sulphanilamide-resistant streptococci can usually be eliminated with propamidine or penicillin, but the latter substance is not yet available for routine use.

Although in some cases it is necossary to employ special drugs, as a general rule raw surfaces can be prepared for grafting by the method already described without additional aids. Success is largely dependent on a careful dressing technique and the prevention of cross-infection.

5. Grafting. Skin replacement is so important in the hand that all raw areas should be grafted as soon as possible, irrespective of their size. Split skin grafts are placed on the raw surfaces and covered with tulle gras, wool and gauze. The dressings are fixed with a crepe bandage and remain in position for about five days. At the end of this

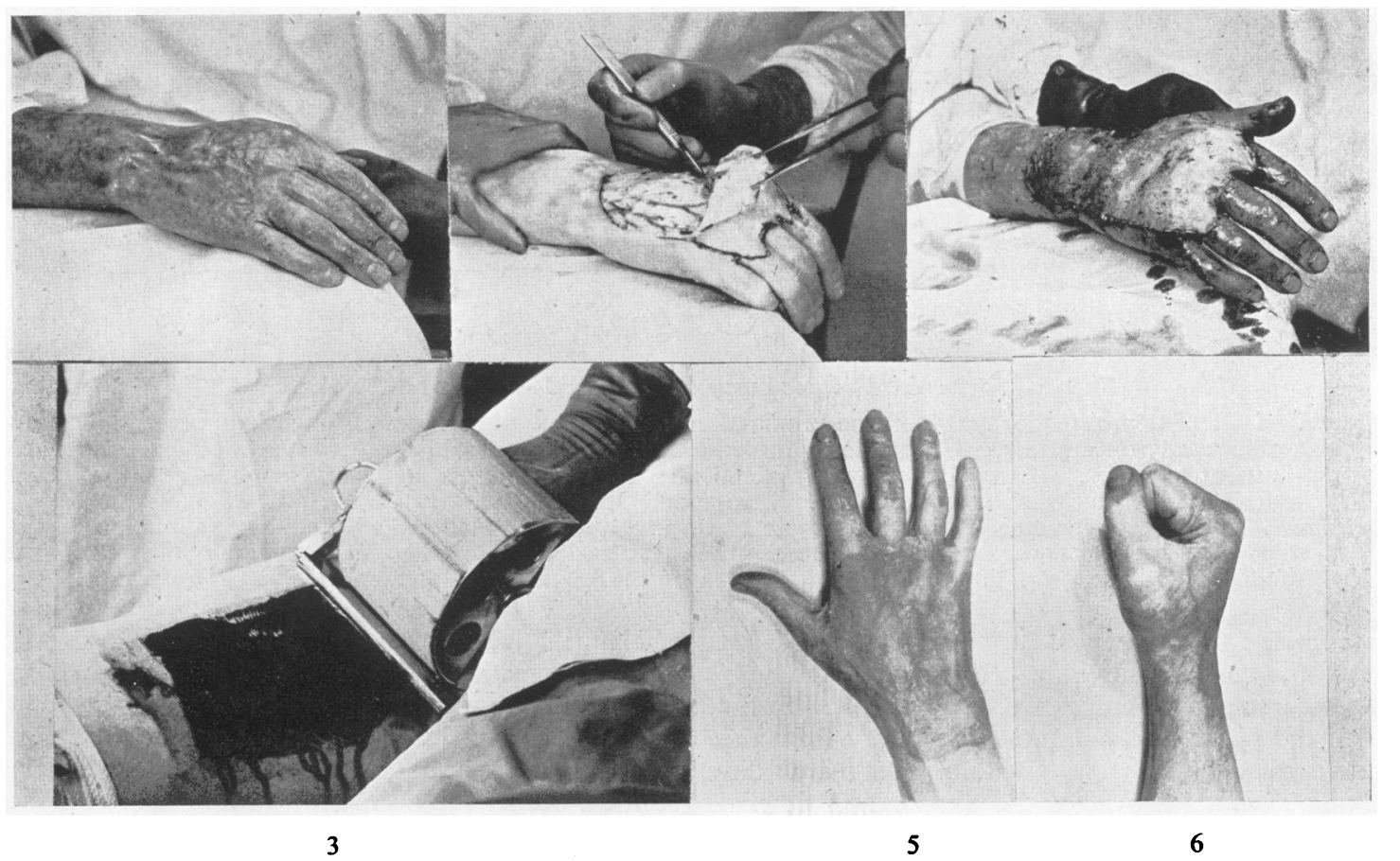

Fig. 2.

1. Healed burn - tight, unstable on dorsum of hand with limitation of movement. 2. Removal of scar.

3. Cutting of graft with Padgett dermatome. 4. Graft sewn in position. 5. and 6. Result-full function. 
time the grafts are inspected and if they have taken successfully healing will be practically complete. The hand is covered for a few more days while the new skin is consolidating, and exercises and active movements are continued.

6. Restoration of Function. A patient with firstor second-degree burns should be able to move his fingers through their full range as soon as the skin is completely healed. If there is residual stiffness it is a serious reflection on the treatment of the case. Limitation of movement at this stage is usually attributable to prolonged and unnecessary immobilization in the preceding period and the surgeon rather than the patient should be blamed.

With third-degree burns, although the primary object is to obtain a completely healed hand, the treatment continues until maximum function is present and the patient is fit to return to work. In some cases an intensive course of physiotherapy is required before the patient regains full power in the hand with proper co-ordination in finger movements. The methods used to help restore function include active exercises, paraffin wax baths, and occupational therapy of a suitable type. Passive movement or manipulation of the fingers is never permitted under any circumstances.

7. Late Surgical Treatment. The surgical treatment of burns of the hands should be considered in two separate phases. In the early period grafts are used to cover raw areas and expedite healing, and in the late stages operation may be required to relieve contractures and improve function. The initial grafting is done in the third or fourth week, but no further operative treatment is usually contemplated until two or three months later. At this stage the case is reviewed and if function is still impaired operation is frequently indicated. Persistent limitation of finger movement is often caused by tight scars and insufficient skin on the dorsum or palm of the hand, and in these cases the patient will be greatly improved by the insertion of a graft. The other indication for grafting is thin, unstable scar tissue at the site of the burn which breaks down on the slightest injury, so that while a full range of movement may be present, incapacity is just as marked as in the cases where there is limitation of movement. Replacement of such unstable scars invariably produces the desired result.

The late operative treatment of the burned hand sometimes includes other procedures such as capsulotomy, arthrodesis, and tendon repair, but space does not permit discussion of these methods.

I am greatly indebted to my teacher Mr. A. H. McIndoe for much help and encouragement in the preparation of this paper. 